\title{
Non-Blocking Concurrent Imperative Programming with Session Types
}

\author{
Miguel Silva \\ Department of Computer Science \\ University of Porto \\ Porto, Portugal
}

\author{
Mário Florido \\ Department of Computer Science \\ University of Porto \\ Porto, Portugal
}

\author{
Frank Pfenning \\ Carnegie Mellon University \\ Pennsylvania, USA
}

\begin{abstract}
Concurrent $\mathrm{C} 0$ is an imperative programming language in the $\mathrm{C}$ family with session-typed messagepassing concurrency. The previously proposed semantics implements asynchronous (non-blocking) output; we extend it here with non-blocking input. A key idea is to postpone message reception as much as possible by interpreting receive commands as a request for a message. We implemented our ideas as a translation from a blocking intermediate language to a non-blocking language. Finally, we evaluated our techniques with several benchmark programs and show the results obtained. While the abstract measure of span always decreases (or remains unchanged), only a few of the examples reap a practical benefit.
\end{abstract}

\section{Introduction}

A session describes the collective conduct of the components of a concurrent system. Binary sessions focus on the interactions between two of these components, with an inherent concept of duality. A session type [9, 10] defines the communication between processes using this notion. Session types enforce conformance to a communication protocol, organizing a session to occur over a communication channel. Recently, session types have been linked with linear logic via a Curry-Howard interpretation of linear propositions as types, proofs as processes, and cut reduction as communication. Variations apply for both intuitionistic [3, 4] and classical [17] linear logic. The intuitionistic variant culminated in SILL, a functional language with session-typed concurrency [16].

The adaptation of SILL to other paradigms of programming gave rise to CLOO (Concurrent Linear Object-Orientation) [2], a concurrent object-oriented language that types objects and channels with session types, and Concurrent $\mathrm{C} 0$ [18], a session-based extension to an imperative language. In this paper, we present a non-blocking model of receiving messages in Concurrent $\mathrm{C} 0$. Our model will be based on the premise that we only want to halt execution to wait for a message when the data contained in that message is necessary to continue computation. We will then present a compilation function from the original version of Concurrent $\mathrm{C} 0$ to our non-blocking model. We also present a cost semantics for Concurrent $\mathrm{C} 0$ that computes the span and work [6], providing an abstract analytical measure of latent parallelism in the computation. The span always improves or remains the same under the non-blocking semantics, although the experimental results show that it is difficult to exploit these improvements in practice.

In related work, Guenot [8] has given a pure and elegant computational interpretation of classical linear logic with non-blocking input based on the solos calculus [11]. The primary notion of a process as a thread of control that pervades our work is no longer visible there and it does not immediately suggest an implementation strategy. Our work generalizes the functional notions of futures and promises [1, 7] by supporting more complex bidirectional communication [15].

I. Cervesato and M. Fernández (Eds): Fourth International Workshop

on Linearity 2016 (LINEARITY'16)

EPTCS 238, 2017, pp. 64-72 doi 10.4204/EPTCS.238.7 
Section 2 briefly introduces the Concurrent $\mathrm{C} 0$ language, outlining its syntax and operational semantics. Section 3 exposes the model for non-blocking reception of messages. Section 4 defines a translation from the original to the non-blocking language. Section 5 provides an experimental evaluation of the implementation of the new model, and Section 6 concludes.

\section{Imperative Programming with Sessions Types}

This section introduces the main concepts of programming of Concurrent $\mathrm{C} 0$ (CC0), a session-based extension of $\mathrm{C} 0$ [13], itself a small safe subset of $\mathrm{C}$ augmented with contracts. For a more in-depth description of $\mathrm{CC} 0$, we refer the reader to a technical report [18].

A program in $\mathrm{CC} 0$ is a collection of processes exchanging messages through channels. These processes are spawned by functions that return channels. The process that calls these spawning functions is called the client. The new process at the other end of the channel is called the provider, who is said to offer a session over the channel.

CC0's session typing system is based on work by Caires and Pfenning [3], who establish a correspondence between session types for $\pi$-calculus and intuitionistic linear logic. CC0's channels have a linear semantics: there is exactly one reference to the channel besides the provider's. This captures the behavior described in the previous paragraph: the process we called the client has the unique reference to the provided channel.

Messages are sent asynchronously: processes advance in parallel without waiting for acknowledgement of the sent message being received. Programmers must specify the protocol of message exchange using session types and a linear type system enforces concordance with this protocol [14].

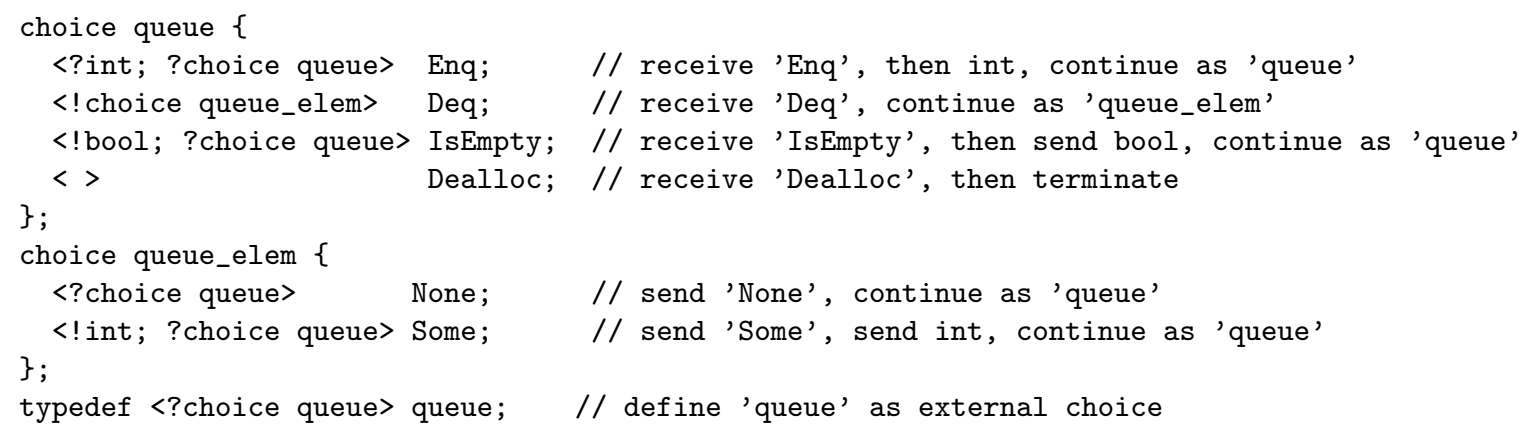

Program 1: Protocol definition of a queue with constant time enqueue and dequeue operations from client's perspective, in $\mathrm{CC} 0$.

Program 1 is an example defining several session types in $\mathrm{CC} 0$. We use $<\ldots .>$ to enclose a session type, ? to indicate an input and ! to indicate and output. An empty $<>$ represents the end of a session. A choice indicates that a label is received (external choice, prefixed by ?) or that a label is sent (internal choice, prefixed by !). Choices have to be explicitly named and declared in $\mathrm{CC} 0$ in a syntax inspired by structs in $\mathrm{C}$.

The queue's protocol exchanges messages in two directions, from the client process to the provider process and vice-versa, resulting in each direction being conveyed independently through an external (queue) and internal choice (queue_elem). The session type is given from the provider's perspective and the compiler determines statically if the operations indicated in the protocol are performend in the correct order according to the channel's session type. 
$\mathrm{CC} 0$ is compiled to a target language and linked with a runtime system, both written in $\mathrm{C}$, responsible for implementing communication.

The session typing of $\mathrm{CC} 0$ uses polarized logic, as detailed in [14], to maintain the direction of the communication. Positive polarity indicates that information is streaming from the provider and negative to the provider. A shift is used to swap polarities. The runtime system explicitly tracks the polarity of each channel and the compiler infers and inserts the minimal amount of shifts into the target language.

$\mathrm{CC} 0$ has the usual features of an imperative programming language, such as conditionals, loops, assignments and functions, extended by communication primitives. Below, we present the core communication syntax of the target language. We differentiate a channel from a variable by placing a $\$$ before the channel name, such as $\$ c$.

\begin{tabular}{|c|c|c|}
\hline$, Q \quad::=$ & $\begin{array}{l}\$ c=\operatorname{spawn}(P) ; Q \\
\$ c=\$ d \\
\operatorname{close}(\$ c) \\
\text { wait }(\$ c) ; Q \\
\operatorname{send}(\$ c, e) ; Q \\
x=\operatorname{recv}(\$ c) ; Q \\
\operatorname{send}(\$ c, \text { shift }) ; Q \\
\text { shift }=\operatorname{recv}(\$ c) ; Q \\
\$ c . l a b ; Q \\
\text { switch }(\$ c)\left\{l a b_{i} \rightarrow P_{i}\right\}_{i}\end{array}$ & $\begin{array}{l}\text { spawn } \\
\text { forward } \\
\text { send end and terminate } \\
\text { receive end } \\
\text { send data (including channels) } \\
\text { receive data (including channels) } \\
\text { send shift } \\
\text { receive shift } \\
\text { send label } \\
\text { receive label }\end{array}$ \\
\hline
\end{tabular}

\subsection{Cost Semantics}

Pfenning and Griffith introduced asynchronous communication using polarized logic [14] in the operational semantics of the target language, which is expressed as a substructural operational semantics [12], based on multiset rewriting [5]. We now extend this operational semantics by assigning a cost to each operation, using the work-span model [6]. We will only count communication costs, ignoring internal computation. Although this may not lead to an entirely realistic measure for the complexity of an algorithm, it will still make for an interesting abstract one. Moreover, in many of our examples communication costs dominate performance.

In the cost semantics we maintain a span $s$ for each executing process which represents the earliest global time (counting only communication steps) at which the process could have reached its current state. Because messages can only be received after they have been sent, each message is tagged with the time at which it is sent, and the recipient takes the maximum between its own span and the span carried by the message. Except for operations using shifts or forwards, each call to a communication function will increase the span by one unit.

The work is determined individually by each process. As with span, all operations except the ones using shifts or forwards will increase work by one unit. Although each message also carries the work of the sending process, this work is ignored unless the message is an end or forward. In these two cases, the receiving process will add the work carried by the message to its own, to propagate the work of the sending process, which is being terminated.

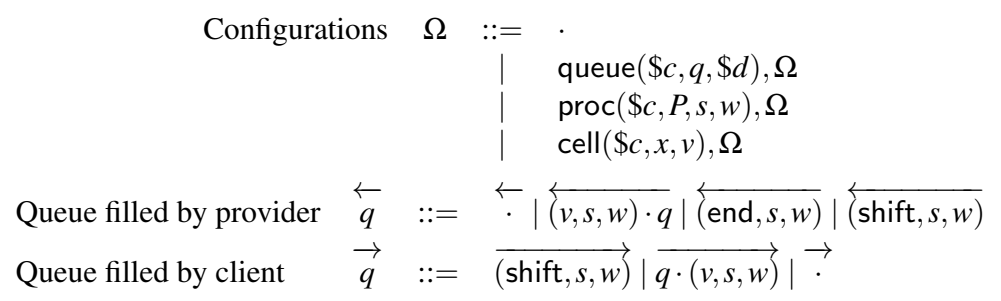


Configurations describe executing processes, message queues connecting processes (one for each channel), and local storage cells. In our definition, $\operatorname{proc}(\$ c, P, s, w)$ is the state of a process executing program $\mathrm{P}$, offering along channel $\$ c$, with span $s$ and work $w$. The message queue is represented by queue $(\$ c, q, \$ d)$, which connects processes offering along $\$ d$ with a client using $\$ c$. The memory cell cell $(\$ c, x, v)$ holds the state of variable $x$ with value $v$ in the process offering along channel $\$ c$. We use the multiplicative conjunction $(\otimes)$ to join processes, queues, and memory cells, and linear implication to express state transition $(\multimap)$. Due to space constraints, we show only some representative rules.

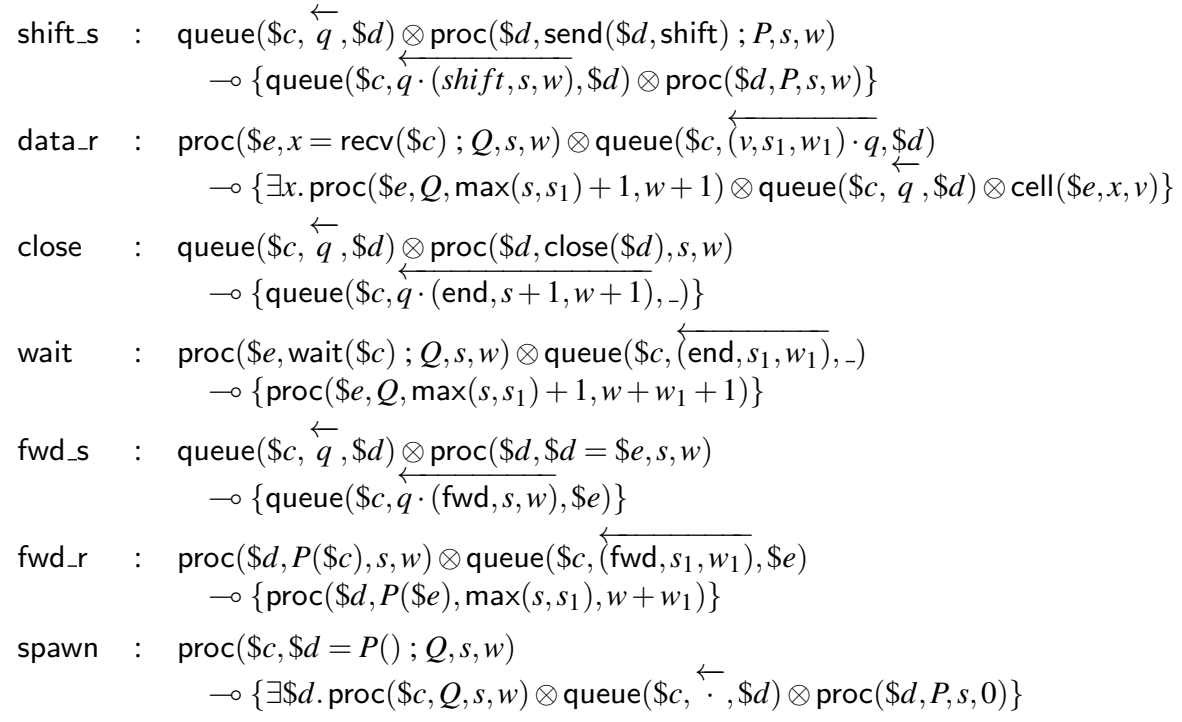

\section{Non-Blocking Receive}

In this section, we present the main contribution of our work: a new model for message reception whose goal will be to block the execution of the process to wait for a message only when the data contained in this message is necessary to continue the execution.

Receiving a message in $\mathrm{CC} 0$ blocks the execution of the program, a behavior matching the operational semantics. A receiving function (recv or wait) will only succeed when it is possible to retrieve the corresponding message from the queue of the associated channel.

This model for message reception may not be the optimal choice for some algorithms where an arbitrary imposed order on messages received on two different channels might prevent other computation to proceed. An extreme case is when a received value is not actually ever needed.

Our alternative for follows two principles. One, the difference should be invisible to the programmer who should not need to know exactly when a message is received. Second, the implementation will still need to adhere to the protocol defined by the session type, which forces the order of sends and receives.

\subsection{Runtime Implementation}

We will now focus on the runtime system, describing how the concepts previously introduced are implemented and how we solved the issues that arose from our model. Our baseline CC0 runtime is implemented in $\mathrm{C}$, using the pthread library in a straightforward way to obtain parallelism in multi-core machines. Message passing is implemented using the queues in shared memory. 
When a process in $\mathrm{CC} 0$ is spawned, it provides a channel to its parent process, i.e., the process that called the spawn function. This channel is represented internally as a structure that aggregates crucial information, namely, a queue of messages.

Our model involves postponing reception as much as possible, by interpreting receives as a request for a message. The request is saved on the channel until a synchronization is necessary. A synchronization is triggered when some data contained on any of the requests is required to continue execution. For example, if a process requested a shift from a channel, a synchronization is required to correct the polarity of the channel, that is, drain the message queue in order to change the direction of communication using the same queue.

The requests are handled in the order they were made, which guarantees that the session type is still being respected. Furthermore, all these changes only occur in an intermediate language, allowing CC0 to keep the same source-level syntax.

From a low level implementation point of view, this change required adding a queue data structure to the channel to hold the requests, creating synchronization functions and modifying the existing receiving functions. It was also necessary to define a translation function between the original, blocking, intermediate language to the new, non-blocking, one. This translation is introduced in the next section. Here are the new constructs introduced by the translation.

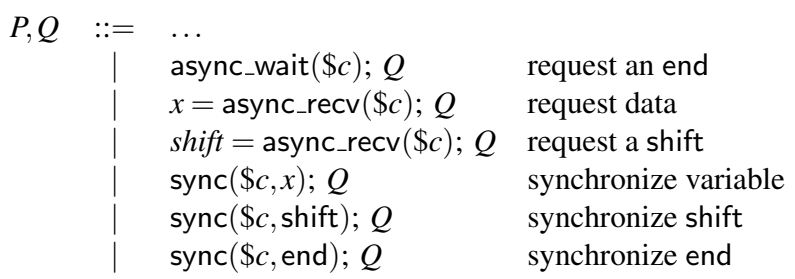

Program 2 shows a possible implementation for the queue example from the previous section. It is presented using an abbreviated version of the target language, using the non-blocking model. Note that receiving a label requires branching between different cases and therefore continues to block in order to avoid speculative execution.

\subsection{Cost Semantics}

We define a receive request to increase both span and work by one unit. Upon synchronising any request, the span must also be synchronized with the value carried by the message. As before, during the synchronization, any end or fwd message will require the addition of the work contained in the message to the work of the receiving process.

There is also the need to change the definition of Configuration, modifying the queue predicate, which will need to keep a new queue of requests, $r$, defined below.

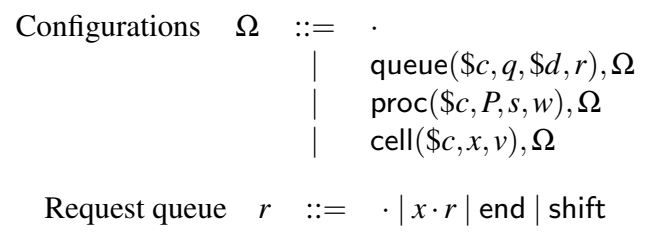

The span of a non-blocking program either decreases or is the same as the corresponding blocking one. For terminating programs, work remains the same between the two models. We have a proof sketch for these two intuitive properties, but a more rigorous proof is still in development. Here, we only show three representative rules. 


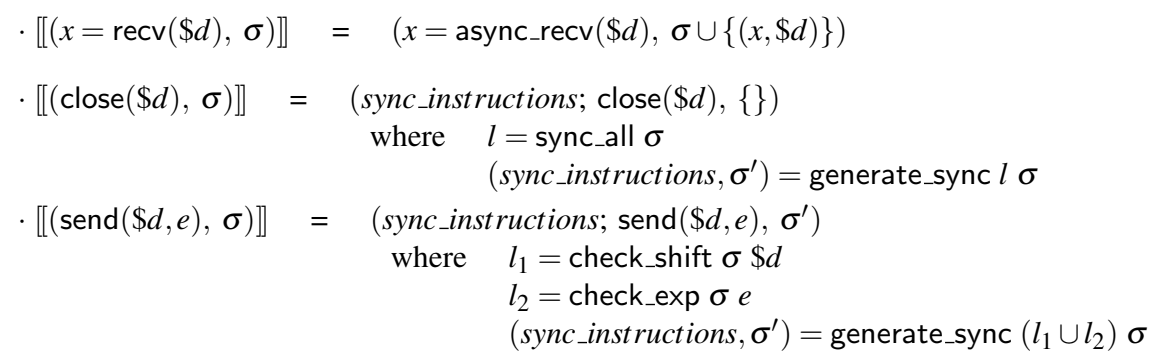

Table 1: Translation scheme of recv-value, close and send-expression operations.

of instructions needed to synchronize the channel. If there is no need to perform any synchronization, generate_sync produces a no operation instruction. This behaviour is captured by the variable sync_instructions: which contains a (possibly empty) list of synchronization instructions "sync( $\$ d$, shift); ...".

To send an expression, we need to perform two different checks: if the channel has the correct polarity and if there is any variable in the expression that needs to be synchronized. The auxiliary functions check_shift and check_exp handle these two verifications, respectively. They return a list of pairs containing the variables and channels that require synchronisation, lists that are passed on to generate_sync, which produces the synchronisation instructions, as mentioned previously. This function also removes all the synchronized pairs from the table of requests.

\section{Experimental Evaluation}

All benchmarks were run on a 2015 Macbook Pro, with a $2.7 \mathrm{GHz}$ Intel Core $\mathrm{I} 5$ ( 2 cores) processor and 8 GB RAM. The benchmarking suite ${ }^{1}$ can be found in table 2, adapted from [18].

\begin{tabular}{|c|c|c|c|}
\hline bitstring1 & bitstrings with external choice & parfib & $\begin{array}{l}\text { parallel naive Fibonacci, simulating } \\
\text { fork/join }\end{array}$ \\
\hline bitstring 3 & bitstrings with internal choice & primes & prime sieve (sequential) \\
\hline & binary search trees, tree sort & queue-notail & queues without tail calls \\
\hline insert-sort & insertion sort using a queue & queue & queues written naturally \\
\hline mergesort1 & mergesort with Fibonacci trees & reduce & reduce and scan on parallel sequences \\
\hline mergesort3 & mergesort with binary trees & seg & list segments \\
\hline mergesort4 & $\begin{array}{l}\text { mergesort with binary trees, se- } \\
\text { quential merge }\end{array}$ & sieve-eager & eager prime sieve merge \\
\hline odd-even-sort1 & odd/even sort, v1 & sieve-lazy & lazy prime sieve \\
\hline odd-even-sort 4 & odd/even sort, v4 & stack & a simple stack \\
\hline odd-even-sort6 & odd/even sort, v6 & & \\
\hline
\end{tabular}

Table 2: CCO benchmarking suite

\footnotetext{
1 available at http://www.cs.cmu.edu/ fp/misc/cc0-bench.tgz
} 


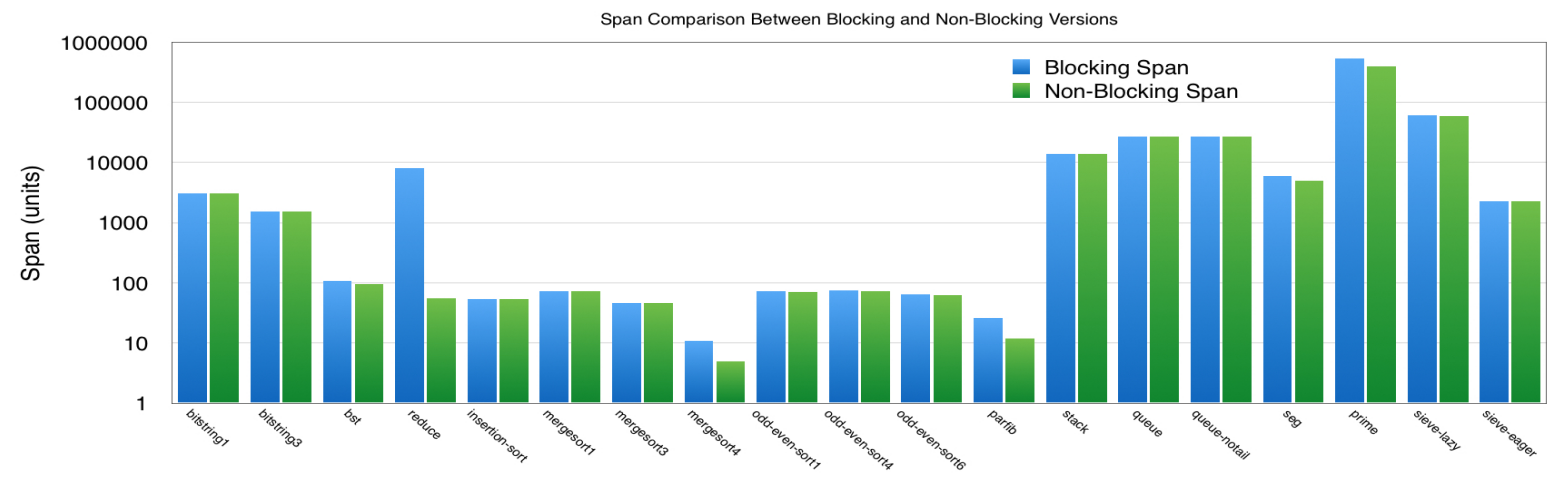

Figure 1: Blocking and Non-Blocking span benchmarks on a log scale.

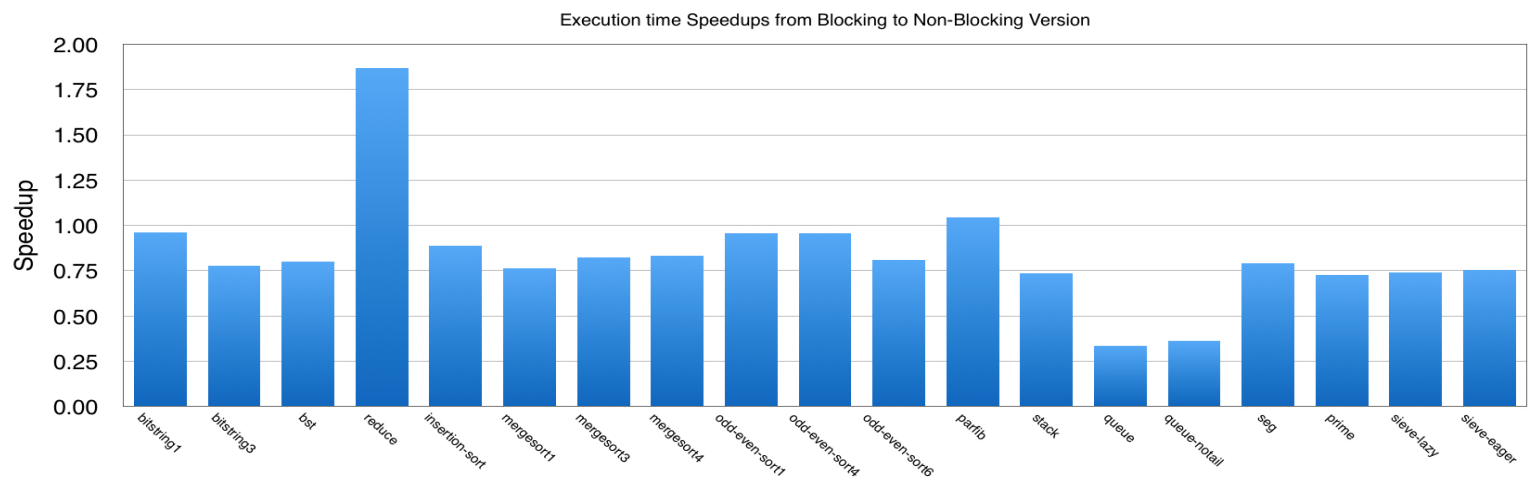

Figure 2: Execution time speedup from Blocking to Non-Blocking Version.

Note that the span is often the same across our benchmark suite, where reduce, mergesort4, and parfib see noticeable improvements. In the case of reduce, some of the difference could be recovered by fine-tuning the $\mathrm{CC} 0$ source program; it is interesting that our generic technique can make up for a performance bug (when considered under the blocking semantics) introduced by the programmer. This shows that, at the very least, our implementation can help identify some performance issues in the given code. As figure 2 shows, the overhead of maintaining the request queue is considerable in some examples. Only in reduce and parfib do we realize an actual performance improvement.

\section{Future Work}

Since recipient behavior on input is opaque to the sender, we may be able to craft an optimisation which avoids the slowdown in the common case where no improvement in the span is available. For this purpose we would likely combine our fully dynamic technique with static dependency analysis to use non-blocking input only where promising. Our cost semantics could also become a tool in performance debugging which may be helpful in particular to novice programmers with little experience in concurrency.

Acknowledgments: This work is partially funded by the FCT (Portuguese Foundation for Science and Technology) through the Carnegie Mellon Portugal Program. 


\section{References}

[1] Henry C. Baker, Jr. \& Carl Hewitt (1977): The Incremental Garbage Collection of Processes. In: Proceedings of the 1977 Symposium on Artificial Intelligence and Programming Languages, ACM, New York, NY, USA, pp. 55-59, doi $10.1145 / 800228.806932$

[2] Stephanie Balzer \& Frank Pfenning (2015): Objects As Session-typed Processes. In: Proceedings of the 5th International Workshop on Programming Based on Actors, Agents, and Decentralized Control, AGERE! 2015, ACM, New York, NY, USA, pp. 13-24, doi:10.1145/2824815.2824817.

[3] Luís Caires \& Frank Pfenning (2010): Session Types as Intuitionistic Linear Propositions. In: Proceedings of the 21st International Conference on Concurrency Theory (CONCUR 2010), Springer LNCS 6269, Paris, France, pp. 222-236, doi $10.1007 / 978-3-642-15375-4 \_16$.

[4] Luís Caires, Frank Pfenning \& Bernardo Toninho (2016): Linear logic propositions as session types. Mathematical Structures in Computer Science 26, pp. 367-423, doi 10.1017/S0960129514000218.

[5] Iliano Cervesato \& Andre Scedrov (2009): Relating state-based and process-based concurrency through linear logic (full-version). Information and Computation 207(10), pp. 1044 - 1077, doi:10.1016/j.ic.2008.11.006 Special issue: 13th Workshop on Logic, Language, Information and Computation (WoLLIC 2006).

[6] Thomas H. Cormen, Clifford Stein, Ronald L. Rivest \& Charles E. Leiserson (2001): Introduction to Algorithms, 2nd edition, chapter 27. McGraw-Hill Higher Education.

[7] Daniel P. Friedman, David \& S. Wise (1978): Aspects of applicative programming for parallel processing. IEEE Transactions on Computers, pp. 289-296, doi 10.1109/TC.1978.1675100

[8] Nicolas Guenot (2014): Session Types, Solos, and the Computational Contents of the Sequent Calculus. Talk at the Types Meeting.

[9] Kohei Honda (1993): Types for Dyadic Interaction. In: 4th International Conference on Concurrency Theory, CONCUR'93, Springer LNCS 715, pp. 509-523, doi:10.1007/3-540-57208-2_35.

[10] Kohei. Honda, Vasco T. Vasconcelos \& Makoto Kubo (1998): Language Primitives and Type Discipline for Structured Communication-Based Programming. In: 7th European Symposium on Programming Languages and Systems, ESOP'98, Springer LNCS 1381, pp. 122-138, doi:10.1007/BFb0053567.

[11] Cosimo Laneve \& Björn Victor (2003): Solos in Concert. Mathematical Structures in Computer Science 13(5), pp. 657-683, doi:10.1017/S0960129503004055.

[12] F. Pfenning \& R. J. Simmons (2009): Substructural Operational Semantics as Ordered Logic Programming. In: Logic In Computer Science, 2009. LICS '09. 24th Annual IEEE Symposium on, pp. 101-110, doi: $10.1109 /$ LICS.2009.8.

[13] Frank Pfenning \& Rob Arnold (2010): CO Language. Available at http://c0.typesafety.net

[14] Frank Pfenning \& Dennis Griffith (2015): Polarized Substructural Session Types. In A. Pitts, editor: Proceedings of the 18th International Conference on Foundations of Software Science and Computation Structures (FoSSaCS 2015), Springer LNCS 9034, London, England, pp. 3-22, doi:10.1007/978-3-662-46678-0_1.

[15] Bernardo Toninho, Luís Caires \& Frank Pfenning (2012): Functions as Session-Typed Processes. In L. Birkedal, editor: 15th International Conference on Foundations of Software Science and Computation Structures, FoSSaCS'12, Springer LNCS, Tallinn, Estonia, pp. 346-360, doi:10.1007/978-3-642-287299_23.

[16] Bernardo Toninho, Luis Caires \& Frank Pfenning (2013): Higher-Order Processes, Functions, and Sessions: A Monadic Integration. In: Proceedings of the 22Nd European Conference on Programming Languages and Systems, ESOP'13, Springer-Verlag, Berlin, Heidelberg, pp. 350-369, doi 10.1007/978-3-642-37036-6_20.

[17] Philip Wadler (2015): Propositions As Types. Commun. ACM 58(12), pp. 75-84, doi:10.1145/2699407.

[18] Max Willsey, Rokhini Prabhu \& Frank Pfenning (2016): Design and Implementation of Concurrent C0. Available at https://www.cs.cmu.edu/ fp/papers/cc016.pdf. 